\title{
Comparing young people's experience of technology-delivered $v$. face-to-face mindfulness and relaxation: two-armed qualitative focus group study
}

\author{
Conall Tunney, Patricia Cooney, David Coyle and Gary O'Reilly
}

\section{Background}

The current popularity of mindfulness-based practices has coincided with the increase in access to mobile technology. This has led to many mindfulness apps and programs becoming available, some specifically for children. However, little is known about the experience of engaging with mindfulness through these mediums.
Aims
To explore children's experience of mindfulness delivered both face-to-face and through a computer game to highlight any differences or similarities.

\section{Method}
A two-armed qualitative focus groups design was used to explore children's experiences. The first arm offered mindfulness exercises in a traditional face-to-face setting with guided meditations. The second arm offered mindfulness exercises through a computer game avatar.

\section{Results}

Themes of relaxation, engagement, awareness, thinking, practice and directing attention emerged from both arms of focus groups. Subthematic codes highlight key differences as well as similarities in the experience of mindfulness.

\section{Conclusions}

These results indicate that mindfulness delivered via technology can offer a rich experience.

\section{Declaration of interest}

C.T., D.C. and G.O.'R. designed the mindfulness program, Mindful gNATS, used in the present study. P.C. is a researcher on another Pesky gNATS game. None of the authors receives any income from Pesky gNATs or Mindful gNATS, which are owned by Handaxe, a community interest company run on a not-for-profit basis.

\section{Copyright and usage}

(c) The Royal College of Psychiatrists 2017.
Mindfulness-based interventions for various mental health difficulties have increased in number and popularity since the introduction of mindfulness-based stress reduction in $1980 .{ }^{1}$ Most notable is mindfulness-based cognitive therapy, ${ }^{2}$ which is recommended by the National Institute for Health and Care Excellence (NICE) ${ }^{3}$ as the treatment of choice for patients with two or more episodes of major depression. However, other psychological interventions, such as acceptance and commitment therapy ${ }^{4}$ and dialectical behaviour therapy, ${ }^{5}$ also include elements of mindfulness. Turning to youth, a narrative review paper by Burke $^{6}$ and the first meta-analysis of mindfulness with youth ${ }^{7}$ tentatively indicate that mindfulness can be an effective component in therapies for a variety of mental health difficulties, anxiety and depression. The growth of mindfulness and the ubiquity of mobile technology and technological interventions has given rise to many online and mobile mindfulness apps for adults and children. As young people and children regularly play games and use apps, these platforms can be convenient mediums from which to support mental health interventions, including mindfulness. As of May 2015 the App Store has 3361 meditation apps and 588 specifically mindfulness apps. The increase in access to technology-based mindfulness instruction and practice is generally regarded as a positive development and could help overcome some of the difficulties young people have with mindfulness, such as boredom. ${ }^{8}$ However, little is known regarding the efficacy, and less still about the experience, of engaging with 'mindful-tech'. There is also a paucity of research regarding whether the experience of mindfulness practice is different when delivered through a technological platform $v$. traditional face-to-face practice.
The aim of this study was to explore children's experiences of mindfulness exercises, delivered both in a face-to-face setting and through a computer game character, to investigate differences or similarities in their experience. This work was undertaken as part of the design of a therapist-assisted in-session cognitivebehavioural therapy (CBT) computer game for children with anxiety or low mood called Pesky gNATs. ${ }^{9}$ In addition to teaching young people core CBT skills, Pesky gNATs trains young people in mindfulness skills, and this content has been used as the basis for a standalone mindfulness-based intervention called Mindful gNATs, ${ }^{10}$ which can be used with both clinical and non-clinical populations to teach mindfulness skills (see online supplement DS1 for a description of these programs). The present study was undertaken with a non-clinical population because of the novel and exploratory nature of the research.

\section{Method}

\section{Study design}

The present study was a two-armed qualitative exploration of young people's experiences of mindfulness exercises. The first arm offered mindfulness exercises in a traditional face-to-face setting with C.T. leading guided meditations. The second arm offered mindfulness exercises led by a computer game avatar, which the children followed. Children did not directly play the game. Focus groups were chosen as the method for data collection as they are considered particularly useful for including the perspective of youth in designing healthcare programs ${ }^{11}$ as well 
as offering data with high face validity as children describe their own view of the world. ${ }^{12}$ A possible drawback of using focus groups with children can be the issue of 'tagging', whereby children agree with previously raised points in preference to raising new ones. Although this intergroup agreement has also been described as increasing the salience of the themes that emerge $^{13}$ it could limit the production of new points of discussion in a focus group. All of the focus groups were single gender as this is considered best practice with young people of this age. ${ }^{14}$ The same moderator ran both arms of focus groups, although the assistant (who attended to recording and running the computer game) varied. Focus groups took place in a classroom in schools, with a maximum of 13 children, and were audio-recorded and transcribed fully before coding. Data collection was carried out in both arms until no new topics related to the experience of mindfulness emerged. Broadly the mindfulness exercises across the two arms were similar, with some variation and were designed to promote awareness of body, mind and world (Fig. 1). Although individual participants did not experience every exercise, the discussion of each exercise ran until a saturation point was reached. Ethical approval for the study was granted by the University College Dublin Human Research Ethics Committee (HS-13-18-Tunney-OReilly) and full written parental consent was obtained for all participants as well as written assent from each young person.

\section{Participants}

In total, 93 children (56\% male) aged 10-12 years (mean 11.07, s.d. $=0.70)$ participated in the study. A convenience sample of children was recruited from fifth and sixth classes (school years seven and eight in the UK, grade six and seven in the USA) in

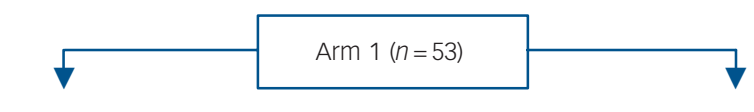

Face-to-face groups

Exercise/skill
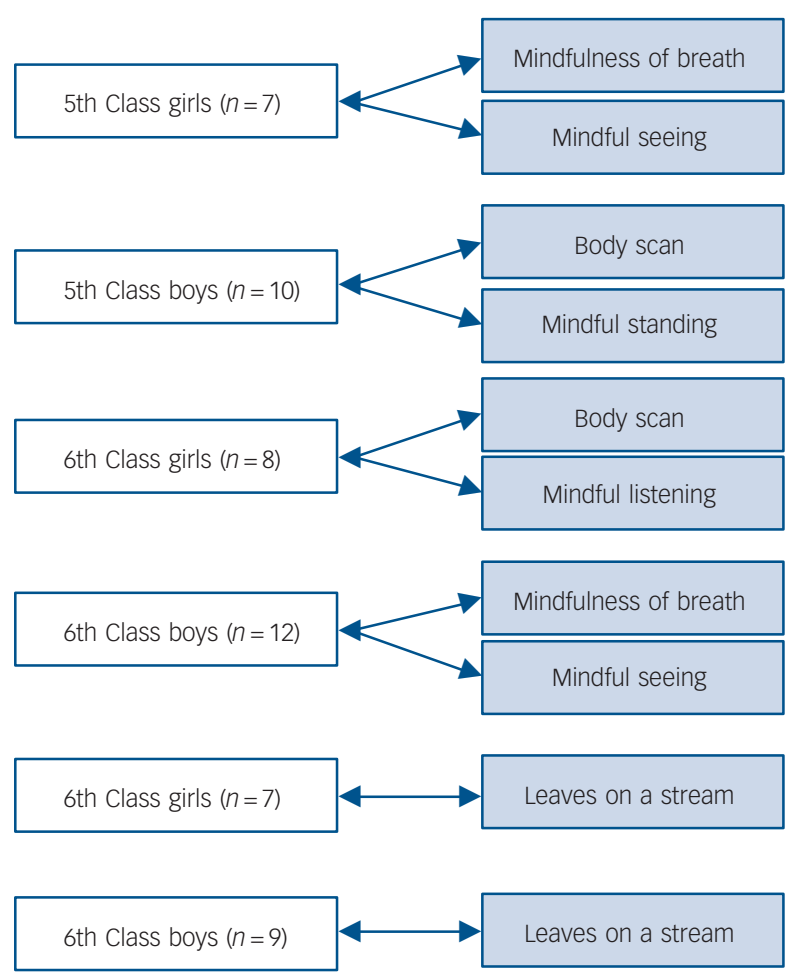

two Irish primary schools. One of the schools was classified as socioeconomically disadvantaged.

\section{Data analysis}

Thematic analysis ${ }^{15}$ was used to identify key themes and subthemes within the data. An exploratory inductive approach was considered appropriate because of the novelty of the topic being investigated. An initial coding frame was produced by C.T. based on emerging clusters of statements. C.T. and P.C. applied this coding frame to a subsection of the data and a discussion of the utility of the frame led to the creation of some new codes and the merging of overlapping codes. The revised coding frame was independently applied to the entire data-set by C.T. and P.C. and 95\% interrater reliability was achieved. It must be considered, however, that both coders were research psychologists with a clinical focus, and being from the same disciplinary background, could therefore have been applying the coding frame with similar approaches to research and perceptions of mindfulness. That said, the percentage agreement observed is very robust. Analysis of codes to produce overarching themes was carried out by C.T. for each of the rounds of focus groups individually. Figures of the themes and codes were then merged to a single graphic model (Fig. 2) to highlight areas of difference and similarity in children's experiences of mindfulness.

\section{Results}

\section{Structure of results}

The thematic map (Fig. 2) illustrates the themes that emerged from the qualitative analysis, as well as the subthematic codes,

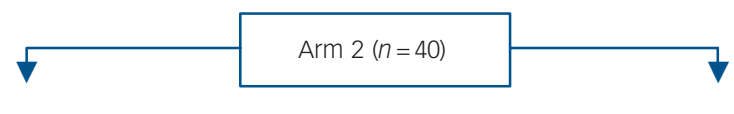

Computerised groups

Exercise/skill
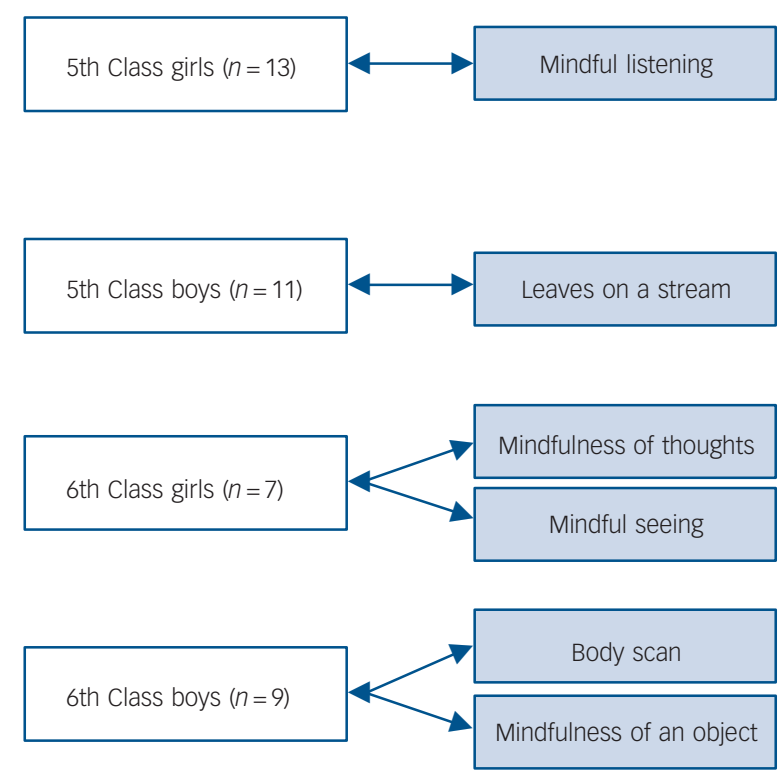


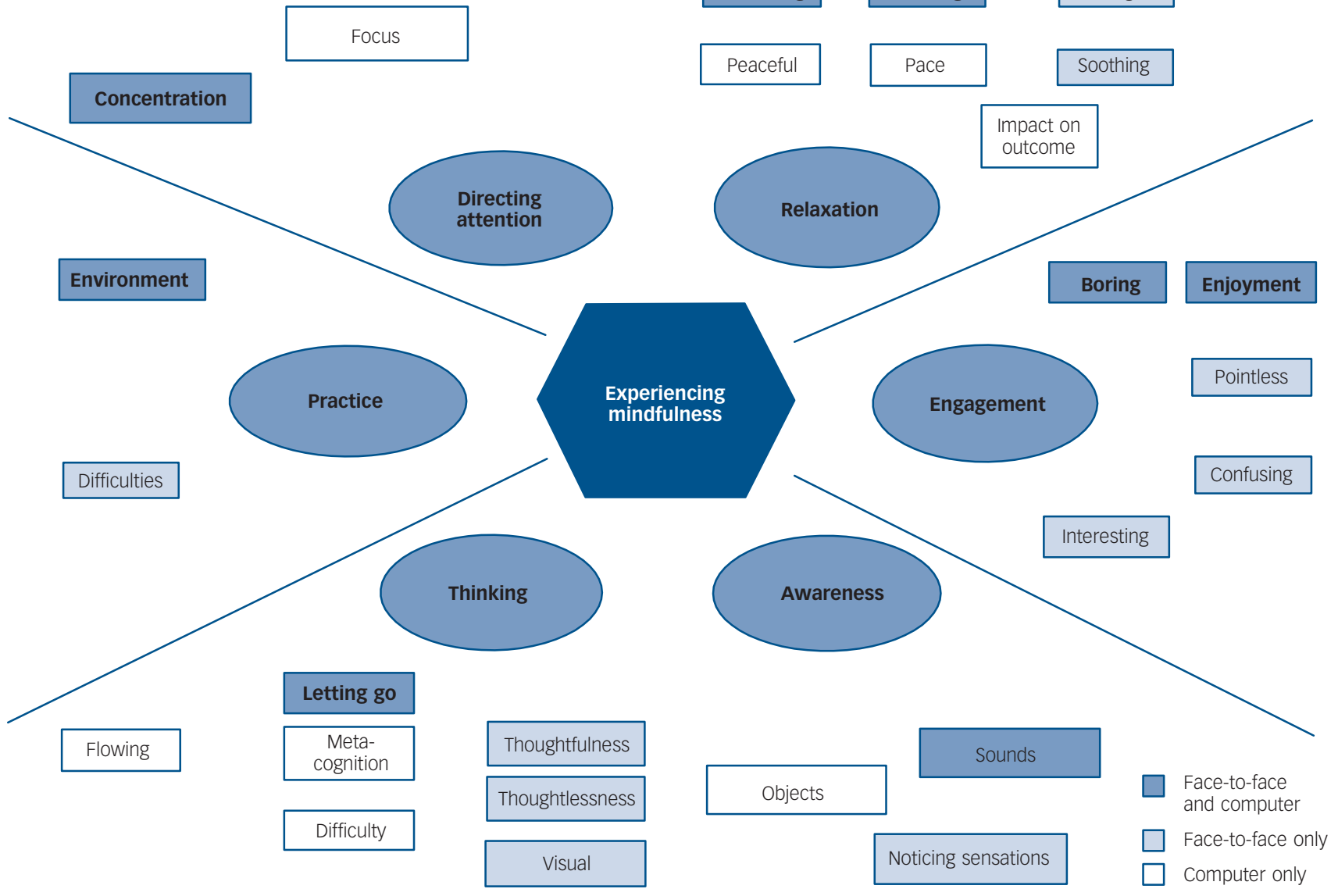

Fig. 2 Themes and codes representing children's experiences of mindfulness both face to face and through a computer game.

which make up the primary themes (shading highlights whether the codes were present in either or both arms of the focus groups). Representative quotes are used to illustrate the context from which codes and themes emerged.

\section{Overview}

Six themes emerged from the analysis of the focus groups: relaxation, engagement, awareness, thinking, practice and directing attention (see online supplement DS2 for a description of themes). All themes were present in the data from both the face-to-face and computerised arms.

\section{Relaxation}

Participants frequently described the feeling of being relaxed and calm while doing the mindfulness exercises. This theme emerged strongly in both face-to-face and computerised focus groups.

'I found them both kinda like calming and relaxing.' (Girl, fifth class - face-to-face group)

'It was peaceful.' (Boy, sixth class - computerised group)

However, the subthematic codes differed somewhat. In the face-to-face focus groups the feeling of being soothed and the physical sensation of becoming tired were discussed by participants.

'It's soothing.' (Girl, fifth class - face-to-face group)

In the computerised groups other dimensions to relaxation were discussed, participants particularly mentioned peacefulness, a slowing down of the pace of their day, and that relaxation was an element that makes engaging in mindfulness possible, or that without relaxation it would be more difficult to actually complete exercises.

'I think when you're trying to let one [thought] go if you're told to eh ... just forget about it and move onto something else, like if you're told, you're not gonna stop thinking about it. But if you just relax for a minute it will just eventually go away.' (fifth class, boy - computerised group)

\section{Engagement}

This theme emerged strongly across both types of focus groups. Participants frequently discussed how they found engaging with the mindfulness exercises. Common experiences across the two platforms were feelings of enjoyment, contrasted by some participants' feelings of boredom.

'I liked it.' (sixth class, boy - face-to-face group)

'I got a bit bored as well,' (sixth class, boy - computerised group)

In the face-to-face groups two codes that contributed to the theme, but were not present in the computerised groups, were descriptions of engaging with the mindfulness exercises as confusing and pointless. In the computerised mindfulness groups another code that contributed to the theme emerged from discussion of the interest participants had in the exercises.

'I like the concept of it.' (fifth class, boy - computerised group)

'Mmm . . . interesting.' (sixth class, girl - computerised group) 


\section{Awareness}

Awareness emerged as a theme across both groups. Participants in both types of group discussed their awareness of the sounds in their environment.

'Kinda felt weird it just felt like I was sitting there and ya can hear the wind.' (sixth class, girl - face-to-face group)

Only participants in the face-to-face groups discussed awareness of physical sensations. They mentioned the sensations of engaging with their environment and noticing their bodies.

'A bit like rough on the carpet.' (fifth class, boy - face-to-face group)

'It looks weird, your chest going up and down.' (sixth class, boy - face-to-face group)

Awareness of specific objects in the surrounding environment was only discussed by participants in the computerised groups.

'You never really think of a book that way you just think here's a book I'll read it.' (sixth class, boy - computerised group)

However, participants' opinion on the usefulness of this awareness was divided.

'Well you wouldn't need to say to people to look at the size of the book cos everyone would see the size when they get it.' (sixth class boy- computerised group)

\section{Thinking}

Thinking emerged as a very strong theme across the two types of groups, although the codes that were identified within each analysis were quite different. The only common subthematic code was the concept of letting go of your thoughts, which was discussed frequently by participants in both groups.

'The way you just have a thought and put it on a leaf and let it flow and move onto the next one.' (fifth class, boy- face-to-face group)

In the face-to-face groups other subthematic codes were thoughtfulness/thoughtlessness, concentration and visualisation. Participants described actively trying to be thoughtful or thoughtless, using mindfulness as a means of concentrating as opposed to daydreaming, and visualising the scenes in the mindfulness exercises.

'It's just like you're getting rid of your thoughts and then some come back to your head and then like go again.' (sixth class, girl - face-to-face group)

'Like if you weren't doing it you'd be daydreaming' (sixth class, boy - face-to-face group)

In the computerised groups the subthematic codes of the flowing nature of thoughts, meta-cognition and difficulty with the exercises emerged.

I like noticing everything that's going on in life at the moment and sometimes your mind can wander off thinking about different things.' (sixth class, girl - computerised group)

'It [letting go of thoughts] like confuses your brain.' (fifth class, girl - computerised group)

\section{Practice}

Participants frequently discussed factors related to practising mindfulness. These factors related to practising mindfulness on the day of the focus groups as well as thinking about practising at home in the future. Within both types of groups participants discussed the role that the practice environment plays in being able to effectively engage with the exercises.

'Distracting [gesturing to the room next door].' (sixth class, girl - face-to-face group)

'Well it depends where you are in your house ... if you're in the sitting room you'd hear the telly.' (fifth class, girl - computerised group)

It was only in the face-to-face groups that participants discussed general difficulties with mindfulness practice.

'I could picture everything but I just didn't get it.' (sixth class, boy - face-to-face group)

\section{Directing attention}

This theme also represents pragmatic discussions by participants in relation to the exercises. In the face-to-face groups concentration was mentioned in terms of the demands placed on them by engaging in the exercises, however, this was not a strong theme in these groups. In the groups offering mindfulness through the computer game character, participants discussed mindfulness itself as a means of concentrating and focusing, and this emerged more strongly that the comparatively brief mention in the face-toface groups.

'It's a way of concentrating.' (fifth class, boy - computerised group)

'It's focusing.' (fifth class, girl - computerised group)

\section{Discussion}

The primary aim of this study was to explore the similarities and differences in children's experiences of mindfulness across two types of delivery, face to face and computerised. To make use of the results in an operational way this discussion will be framed in the context of how well the face-to-face experience of mindfulness carries over to the computerised experience, i.e. is the computerised platform a comparable and valid means of delivering mindfulness. Then, a comparison of these results to the existing research on children's experiences of mindfulness will contextualise this paper within the current body of literature. Finally, we will discuss whether the experience of mindfulness from the computerised focus groups maps onto the conceptualisation of mindfulness used in Mindful gNATs (see online supplement DS1).

\section{Main interpretation}

The primary findings of this study are the consistent overlap of themes across the face-to-face and computerised experiences of mindfulness, and the differences and similarities in the subthematic codes that make up the primary themes. The key themes of relaxation, engagement, awareness, thinking, practice and directing attention emerged from the analysis of each arm of focus groups. This finding is an indication that the experience of engaging in mindfulness exercises delivered by a computer avatar is thematically or broadly similar to the experience when a person delivers the exercises face to face. Further, this result suggests that the 'active ingredient', and potential mechanism of change, of the mindfulness exercises in this study is associated with the content of the exercises themselves and not with the human contact of being guided by a person. Although this overall finding, in terms of themes, is a strong indication that a technological platform is a valid means of delivering mindfulness, the subthematic codes highlight the important differences in the experience that were also observed in the results.

Within the themes of engagement and practice there are codes that provide insight into the practical experience of engaging with the mindfulness content. In both arms of focus groups there were some children who discussed 'enjoying' the mindfulness and some who described their experience as 'boring'. However, some children in the face-to-face groups discussed being 'confused' by the exercises and some considered them 'pointless'. These codes did not arise in the computerised groups where children also talked about their interest in the computer program and the exercises. Within the theme of practice, a code for 'general difficulty' with the mindfulness content emerged from the faceto-face groups and, although the difficulty of the content in both groups was at the same level, no similar code emerged from the computerised groups. The fact that the computerised content was supported by a visual interface throughout the exercises, as 
opposed to a mostly auditory delivery in the face-to-face groups, could account for this difference. In general, these subthematic codes lend support to the computerised delivery of these exercises.

Within the theme of relaxation, which emerged strongly in both sets of groups, there were distinct differences in the subthematic codes. Conversations about the experience being 'soothing' and 'tiring' were observed in the face-to-face groups but not the computerised groups. It is possible that the human element in the delivery of the face-to-face groups created a more soothing experience. Although this is a positive aspect of the experience the authors speculate whether the higher level of contentment associated with being soothed could also lead to more tiredness, which could impede engagement with the content. 'Pace', 'peaceful' and 'impact on outcome' were all codes that emerged from the computerised groups only. Children discussed the change in pace to their day when the computer avatar began to teach and direct mindfulness. This difference in pace, which young people only noted in the computerised groups, could be a factor that is more pronounced for mindfulness in technology. In traditional mindfulness teaching, the leader gauges and engages the group in a natural way. The inability of technology to do this may have implications for mindfulness practice and is an element of the experience that future qualitative research could explore. Children in the computerised groups also discussed peacefulness, a code that was linked to ambient sounds such as bird noises that are built into the game. Finally, the need for relaxation so that they could engage with the exercises was discussed by children in the computerised groups. This description of the role that relaxation plays in meditation indicates that the teaching in Mindful gNATs offers children a good understanding of meditation even in a brief exposure.

A final area of the data that offers a deeper picture of children's experience is around the cognitive factors that were discussed. 'Letting go' of thoughts was the only common code across both sets of groups. Being deliberately 'thoughtful' or 'thoughtless', 'concentration' and 'visualisation' were discussed in the face-toface groups only, whereas 'meta-cognition', 'difficulty' and 'flowing thoughts' were discussed in the computerised groups only. Children in the face-to-face groups described engaging with their thoughts as they meditated, children in the computerised groups described thinking about their thinking as they meditated.

Previous research has demonstrated the feasibility and acceptability of mindfulness content for children and adolescents, when delivered in a face-to-face format, with a variety of presenting difficulties as well as young people without a current difficulty. ${ }^{16}$ Semple et al ${ }^{16}$ carried out a small-scale qualitative study with children aged 5-6 and observed a high level of enjoyment of face-to-face mindfulness. Mendelson et a l $^{17}$ demonstrated acceptability of school-based face-to-face mindfulness with a large sample of elementary school children. Studies with adolescents, which looked at the acceptability of face-to-face mindfulness, also observed positive results with high school, primary care and socially disadvantaged populations. ${ }^{18-20}$ This study builds on these findings and our results suggest a high level of feasibility and acceptability of computerised mindfulness for school-based populations.

Many elements of the mindfulness experience described by children in this study map onto the findings of previous research that included qualitative elements in their designs. Broderick \& Metz ${ }^{18}$ reported the feelings of tiredness described by participants in their study, and observed that those who practised mindfulness most often had the highest levels of tiredness. Tiredness only emerged in the face-to-face arm of the present study. There may be potential for technology to decrease the level of tiredness experienced by young people practising mindfulness. Monshat et $a l^{20}$ identified relaxation and calm, not being controlled by emotions, developing a mindfulness practice routine and learning to take a considered stance towards the self as factors that adolescents discussed about mindfulness. The themes of relaxation and calm and practising mindfulness are represented strongly in our findings. The other themes observed by Monshat et al ${ }^{20}$ are less linked to the simple present moment awareness model of mindfulness in Mindful gNATs and were not observed in the present study.

Quantitative studies on mindfulness with young people have identified positive effects of various face-to-face mindfulnessbased programmes for attention, ${ }^{21-23}$ emotional regulation, ${ }^{24}$ meta-cognition and executive functioning, ${ }^{25}$ and anxiety and relaxation. ${ }^{26,27}$ Using an inductive analytic approach the present study identified many codes that indicate the mindfulness content in Mindful $g N A T s^{10}$ facilitates an experience that matched these areas.

The Mindful gNATs exercises aim to facilitate non-judgemental awareness of the body, mind and world in the present moment. In order to evaluate whether it was successful in providing an experience of mindfulness consistent with this goal, a deductive approach must be adopted. Awareness of the environment and of thoughts can be seen in the themes of awareness and thinking. This result indicates that the program was successful in facilitating awareness of these areas for young people. However, specific discussion around noticing the body was not observed in the computerised arm of focus groups, even though there were exercises aimed at this type of awareness. Although this was a surprising result, overall the results support the efficacy of Mindful $g_{N A T s}{ }^{10}$ for promoting awareness of the environment and the mind. Future research with the program should explore its potential to promote awareness of the body further.

\section{Strengths and limitations}

As a qualitative design was utilised in this study the sample size accessed is robust, and allowed results to be analysed in separate categories while maintaining a large sample in each category. A key strength in this study is the high level of interrater reliability achieved in the thematic analysis. Although this is largely a result of the simplicity and coherence of the data, it allows for stronger conclusions to be drawn from the findings. The recruitment of schools in different socioeconomic brackets offers strength to the findings by accessing a diverse sample of the target population for the program. However, the sample was a convenience sample drawn from the same city, in urban areas, with ready access to high-speed internet and better technological infrastructure. This may limit the generalisability of the findings to populations from rural areas

The fact that all participants did not engage with the same exercises could be regarded as a weakness in the study design. However, this is offset by the large sample size and the fact that focus groups ran until a level of saturation in the discussion was reached. Further, not all exercises in the face-to-face arm were the same as those in the computerised arm. This was because of the need to amend the exercises for the technological platform. Although all the exercises were not exactly the same, the same three areas of awareness were being targeted in both arms, which alleviates the impact of this difference.

\section{Future research}

Future studies should seek to control for participants' levels of prior experience with both technology and with mindfulness, or other forms of meditation, to strengthen their samples. Although 
the results of this study, for both face-to-face and computerised groups, could be analysed under the same thematic headings the present study highlighted the key differences and similarities in the experience of mindfulness delivered across the two platforms. An important avenue for future research is to explore those differences, particularly as more therapeutic content is being delivered via technology. Further, research using a similar design to the present study, but with a clinical population, could indicate whether these differences and similarities are also pertinent to populations currently experiencing a mental health difficulty. In order to advance the research on the specific topic of the present study a quantitative trial, which builds on the experience of mindfulness reported by young people, is needed. Standardised measures, which reflect the themes of relaxation, awareness and thinking, should form the basis of a randomised controlled trial. Further, task-based assessments evaluating codes that could be regarded as skills, such as meta-cognition, would inform how mindfulness is operationalised by young people.

\section{Implications}

The results of this study strongly indicate that mindfulness delivered via technology can offer a rich experience of this type of meditation. Broadly speaking, if the same exercises are delivered face to face or via a computer game avatar a similar general experience can be expected for participants. However, the elements that make up the experience are likely to differ as follows. Face-to-face mindfulness is a more soothing and tiring experience, but computerised mindfulness has a greater impact on pace of life and peacefulness, and is preferable to children. Further, whereas face-to-face delivery is a cognitively active experience, computerised delivery promotes higher levels of meta-cognition. In conclusion, computerised delivery potentially offers an effective, rich and enjoyable mindfulness experience that mental health professionals could add to their practice.

Conall Tunney, PhD, Patricia Cooney, BA, School of Psychology, University College Dublin, Dublin, David Coyle, MSC, PhD, School of Computer Science, University College Dublin, Dublin; Gary O’Reilly, MA, MPsychSc, PhD, School of Psychology, University College Dublin, Dublin, Ireland

Correspondence: Conall Tunney, School of Psychology, University College Dublin, Belfield, Dublin 4, Ireland. Email: conall.tunney@ucdconnect.ie

First received 20 Jul 2015, final revision 30 Jun 2016, accepted 1 Jul 2016

\section{References}

1 Kabat-Zinn J. Outpatient program in behavioural medicine for chronic pain patients based on the practice of mindfulness meditation: theoretical considerations and preliminary results. Gen Hosp Psychiatry 1982; 4: 33-47.

2 Segal ZV, Williams JMG, Teasdale JD. Mindfulness-based Cognitive Therapy for Depression: A New Approach to Preventing Relapse. Guilford Press, 2002.

3 National Institute for Health and Care Excellence. Scope: Depression in Children (FINAL with Amendment). NICE, 2003 (https://www.nice.org.uk/ guidance/cg28/documents/scope-depression-in-children-4).
4 Hayes SC, Strosahl K, Wilson KG. Acceptance and Commitment Therapy: An Experiential Approach to Behavior Change. Guilford Press, 1999.

5 Linehan MM. Cognitive Behavioural Therapy of Borderline Personality Disorder. Guilford Press, 1993.

6 Burke CA. Mindfulness based approaches with children and adolescents: a preliminary review of research in an emergent field. J Child Fam Stud 2010; 19: $133-44$.

7 Zoogman S, Goldberg SB, Hoyt WT, Miller L. Mindfulness intervention with youth: a meta-analysis. Mindfulness 2015: 6; 290-302.

8 Thompson M, Gauntlet-Gilbert J. Mindfulness with children and adolescents: effective clinical application. Clin Child Psychol Psychiatry 2008; 13: 395-407.

9 O' Reilly G, Coyle D. Pesky gNATS Cognitive Behaviour Therapy Computer Game. Handaxe CIC, 2015 (www.PeskyGnats.com).

10 O' Reilly G, Coyle D. Mindful gNATs App. Handaxe CIC, 2015 (http:// www.mindfulgnats.com/).

11 Peterson-Sweeney $\mathrm{K}$. The use of focus groups in pediatric and adolescent research. J Pediatr Health Care 2005; 19: 104-10.

12 Heary CM, Hennessy E. The use of focus group interviews in pediatric health care research. J Pediatr Psychol 2002; 27: 47-57.

13 Lewis A. Group child interviews as a research tool. Brit Educ Res J 1992; 18: 413-21.

14 Stewart DW, Shamdasani PN, Rook DW. Sage Research Methods. Sage, 2007.

15 Braun V, Clarke V. Using thematic analysis in psychology. Qual Res Psychol 2006; 3: 77-101.

16 Semple RJ, Reid EFG, Miller L. Treating anxiety with mindfulness: an open trial of mindfulness training with anxious children. J Cogn Psychother 2005; 19: 379-92.

17 Mendelson T, Greenberg MT, Dariotis JK, Gould LF, Rhoades BL, Leaf PJ. Feasibility and preliminary outcomes of a school-based mindfulness intervention for urban youth. J Abnorm Child Psychol 2010; 38; 985-94.

18 Broderick PC, Metz S. Learning to BREATHE: a pilot trial of a mindfulness curriculum for adolescents. Adv Sch Ment Health Promot 2009; 2: 35-46.

19 Sibinga EMS, Stewart M, Magyari T, Welsh CK, Hutton N, Ellen JM. Mindfulness-based stress reduction for HIV-infected youth: a pilot study. Explore (NY) 2008; 4: 36-7

20 Monshat K, Khong B, Hassed C, Vella-Broderick D, Norrish J, Burns J, et al. "A conscious control over life and my emotions:" mindfulness practice and healthy young people. A qualitative study. J Adolesc Health 2013; 5: 57-77.

21 Semple RJ, Lee J, Rosa D. A Randomised trial of mindfulness-based cognitive therapy for children: promoting mindful attention to enhance social-emotional resiliency in children. J Child Fam Stud 2010; 19: 218-99.

22 van de Oord S, Bogels SM, Peijnenburg D. The Effectiveness of mindfulness training for children with ADHD and mindful parenting for their parents. J Child Fam Stud 2012; 21: 139-147.

23 Napoli M, Krech PR, Holley LC. Mindfulness training for elementary school students. J Appl Sch Psychol 2005; 21: 99-125.

24 Schonert-Reichl KA, Lawlor MS. The effects of a mindfulness-based education program on pre- and early adolescents' well-being and social and emotional competence. Mindfulness 2010; 1: 137-51.

25 Flook L, Smalley SL, Kitil MJ, Galla BM, Kaiser-Greenland S, Locke J, et al. Effects of mindful awareness practices on executive functions in elementary school children. J Appl Sch Psychol 2010; 26: 70-95.

26 Haydicky J, Weiner J, Badali P, Milligan K, Duchame JM. Evaluation of a mindfulness-based intervention for adolescents with learning disabilities and co-occurring ADHD and anxiety. Mindfulness 2012; 3: 151-64.

27 Biegel GM, Brown KW, Shapiro SL, Schubert CM. Mindfulness-based stress reduction for the treatment of adolescent psychiatric outpatients: a randomised clinical trial. J Consult Clin Psychol 2009; 77: 855-66. 